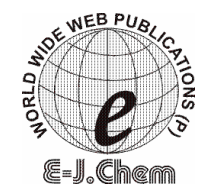

http://www.e-journals.net

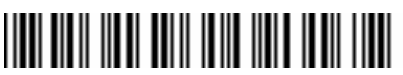

ISSN: 0973-4945; CODEN ECJHAO

E-Journal of Chemistry

Vol.5, No.1, pp. 68-73, January 2008

\title{
Isolation and Characterization of Process-Related Impurity in Azoxystrobin
}

\author{
S. G. HIRIYANNA*, K. BASAVAIAH and K. SREEDHAR ${ }^{\#}$ \\ *Department of Chemistry, \\ Manasagangothri, University of Mysore, Mysore-570009, India. \\ "Department of Chemistry, \\ Kakathiya University, Warrangal, AP, India. \\ hiriyannag@gmail.com
}

Received 26 April 2007; Accepted 31 May 2007

\begin{abstract}
One unknown impurity in azoxystrobin bulk material at a level $0.15 \%$ was detected by a gradient reverse phase high performance liquid chromatography (HPLC). This impurity was isolated from a crude sample of azoxystrobin using reverse phase preparative LC. The impurity isolated by preparative LC was characterized by NMR, MS experimental data. Based on the results obtained from different spectroscopic experiments, these impurity was characterized as methyl 2(2- (6- (2-cyanophenoxy)-2-((4-(2-cyanophenoxy)-6- (2- (1, 3-dimethoxy-3oxoprop-1-en-2-yl)phenoxy) pyrimidin-5-yl) methyl)pyrimidin-4-yloxy)phenyl)3-methoxyacrylate. Formation of this impurity is also discussed.
\end{abstract}

Keywords: Azoxystrobin; Impurity; Preparative LC, Spectroscopy; Isolation; Identification and Characterization.

\section{Introduction}

Azoxystrobin, methyl 2-(2-(6-(2-cyanophenoxy)pyrimidin-4-yloxy)phenyl-3-methoxy acrylate is a potent antifungal agent ${ }^{1-3}$. High performance liquid chromatographic (HPLC) method was developed for testing of purity of azoxystrobin bulk material. The stringent purity requirement from regulatory agencies ${ }^{5}$ that all the individual impurity, which are $\geq$ $0.1 \%$, must be identified and characterized, this paper aims at the identification and characterization of an unknown impurity in azoxystrobin active ingredient by LC-MS/MS and $\mathrm{NMR}^{4}$. To the best of our knowledge, this impurity is novel, and not reported in literature. Since the impurity level is low, after trying several days, failed to enrich the impurity level by synthetic route. A thorough study has been undertaken to develop a preparative LC method to isolate, wherever possible, and characterize this impurity by chromatographic and spectroscopic techniques. 


\section{Experimental}

\section{Samples and chemicals}

The samples of bulk azoxystrobin were received from Advinus Agro Research Cntre, Bangalore, India. HPLC grade acetonitrile was obtained from Merck Co., Mumbai, India. HPLC grade Acetone was obtained form Rankem, Mumbai, India. Ultra pure water was collected from Elix Millipore water purification system. HPLC grade Dichloromethane used for liquid liquid extraction was purchased from Spectrochem, Mumbai, India. Nitrogen and Zero Air used were of ultra pure grade $(99.999 \%)$.

\section{$H P L C$}

An Agilent HPLC system equipped with 1200 series low pressure quaternary gradient pump along with pulse dampener, photo diode array detector and auto liquid sampler handling system has been used for the analysis of samples. The data was collected and processed using Agilent "Chemstation" software. An Inertsil ODS-3V (250* $4.6 \mathrm{~mm}, 5$-Micron, GL Sciences, Japan) column was employed for the separation of impurity from azoxystrobin. The column eluent was monitored at detection wavelength $220 \mathrm{~nm}$. A linear gradient programme was optimized for the clear separation of impurity from azoxystrobin active ingredient where the initial mobile phase ratio was a mixture of $0.04 \% \mathrm{v} / \mathrm{v}$ orthophosphoric acid and acetonitrile in a ratio of 50:50, v/v for $10 \mathrm{~min}$. Subsequently the percentage of acetonitrile in the mobile phase was increased from 50 to 90 up to $25 \mathrm{~min}$. The same ratio was held for $20 \mathrm{~min}$, and brought back to initial condition within $5 \mathrm{~min}$. The column was allowed to get equilibrated for $10 \mathrm{~min}$ before performing the next injection. Chromatography was performed at room temperature using at a flow rate of $1.0 \mathrm{~mL} \mathrm{~min}^{-1}$

\section{High performance liquid chromatography (preparative)}

An Agilent preparative HPLC system equipped with 1200 series pump, photo diode array detector, auto liquid sampler handling system fitted with $900 \mu \mathrm{L}$ loop and 1200 series preparative fraction collector has been used. The data was collected and processed using Agilent "Chemstation" software. A Zorbax C18 column (50 X 50 mm, 5-Micron, Agilent Technologies) was employed for loading the sample. An analytical method was developed in isocratic mode separately to resolve this impurity, followed by scaling up the same method for preparative HPLC to collect the required impurity fractions. The mobile phase consisted of water acetonitrile in the ratio of $25: 75(\mathrm{v} / \mathrm{v})$. The flow rate was set at $25 \mathrm{~mL} \mathrm{~min}^{-1}$. Detection was carried out at 220 $\mathrm{nm}$. Approximately $200 \mathrm{mg} \mathrm{mL}^{-1}$ of sample was prepared using acetonitrile and water as diluent in the ratio of 50:50,v/v to load on to the column.

\section{Mass spectrometry ( $L C-M S / M S$ )}

LC-MS/MS analysis has been performed on API 2000, Mass Spectrometer (Applied Biosystems). The analysis was performed in positive ionization mode with turbo ion spray interface. The parameters for Ion source voltage IS $=5500 \mathrm{~V}$, declustering potential, $\mathrm{DP}=70 \mathrm{~V}$, focusing potential , $\mathrm{FP}=400 \mathrm{~V}$, entrance potential, $\mathrm{EP}=10 \mathrm{~V}$ were set with nebuliser gas as air at a pressure of $40 \mathrm{psi}$ and curtain gas as nitrogen at a pressure of 25 psi. An Inertsil ODS-3V (250*4.6 mm, 5-Micro, GL Sciences, Japan) column was used for the separation. The mobile phase is a mixture of water, acetonitrile in a ratio of 50:50 (v/v). The analysis was performed at a flow rate of $1.0 \mathrm{~mL} \mathrm{~min}^{-1}$.

\section{NMR spectroscopy}

The ${ }^{1} \mathrm{H}$ and ${ }^{13} \mathrm{C}$ NMR experiments of one of the impurity were carried out at a frequencies of 200 $\mathrm{MHz}$ and $50 \mathrm{MHz}$ respectively, in $\mathrm{CDCl}_{3}$ at $25^{\circ} \mathrm{C}$ temperature on a Bruker Avance dpx- $200 \mathrm{FT}$ 
NMR spectrometer. ${ }^{1} \mathrm{H}$ and ${ }^{13} \mathrm{C}$ chemical shifts are reported on the $\delta$ scale in ppm, relative to tetra methyl silane (TMS) $\delta 0.00$ and $\mathrm{CDCl}_{3}$ at $77.0 \mathrm{ppm}$ in ${ }^{13} \mathrm{C}$ NMR respectively. $\mathrm{D}_{2} \mathrm{O}$ exchange experiment was performed to confirm the exchangeable protons.

\section{FT IR spectroscopy}

IR spectra of the impurity of azoxystrobin was recorded in the solid state as $\mathrm{KBr}$ dispersion using Perkin Elmer spectrum 100 FT-IR spectrophotometer with DRS (Diffuse Reflectance Sampler) accessory.

\section{Results and Discussion}

\section{Detection of impurity by HPLC}

Typical analytical LC chromatograms of azoxystrobin and its impurity obtained by using the LC method discussed in the experimental section are shown in Figure 1. The targeted impurity under study are marked as Impurity I eluted at retention times of about 23 min and azoxystrobin eluted at about $18 \mathrm{~min}$. Crude sample of azoxystrobin was also injected to check the level of impurity.
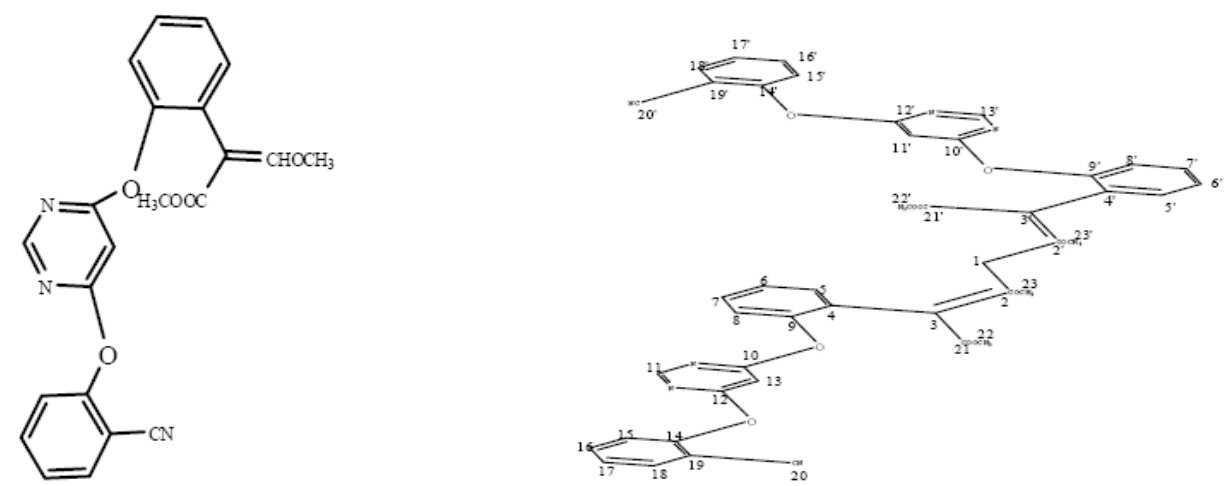

Figure 1. Chemical structures of azoxystrobin and impurity I

\section{LC-MS/MS analysis}

LC-MS/MS analysis of crude sample of azoxystrobin was performed using the solvent system as described in the experimental section. Results of LC-MS/MS analysis revealed that the impurity exhibited molecular ion at $\mathrm{m} / \mathrm{z}(\mathrm{M}+1) 819$ amu and mass fragmentation was carried for this molecular ion. Mass fragmention parrtern of impurity I supports the attachement of $-\mathrm{CH}_{2}$ - in the position of 2, 2' marked in the chemical structure of impurity I (refer Figure 1). To unambiguously confirm the structure of the impurity $(\mathrm{m} / \mathrm{z} 819, \mathrm{M}+1)$, this impurity was isolated by preparative HPLC in a pure form.

\section{Isolation of the impurity $(\mathrm{m} / \mathrm{z} ; \mathrm{819})$ by preparative HPLC}

A simple reverse phase solvent system discussed in the experimental section was used for isolating the impurity with $\mathrm{m} / \mathrm{z}$ 819(M+1)amu . In this solvent system, azoxystrobin eluted at about $11.5 \mathrm{~min}$ whereas the impurity eluted at about $15.0 \mathrm{~min}$. The impurity fractions isolated, were concentrated by removing acetonitrile layer at room temperature under high vacuum on a Buchii Rotavapour Model R124. The aqueous layer containing this impurity was extracted into methylene chloride using the separating funnel. The methylene chloride layer which is having impurity is dried through anhydrous sodium sulphate. These fractions were concentrated at room temperature under high vacuum on a Rotavapour. Purity of this impurity was tested in analytical mode and was found to be $99.4 \%$ before carrying out spectroscopic experiments. 


\section{Structural elucidation of impurity}

LC-MS/MS spectral data of impurity I was compared with the spectral data of azoxystrobin. This impurity exhibited molecular ion at $\mathrm{m} / \mathrm{z} 819(\mathrm{M}+1)$ in LC-MS/MS analysis. This molecular ion is exactly $14 \mathrm{amu}$ more than that of azoxystrobin dimer. The impurity is formed as a dimmer with an addition of methylene group. The mass fragmentation pattern of impurity I is shown in the Figure 2.

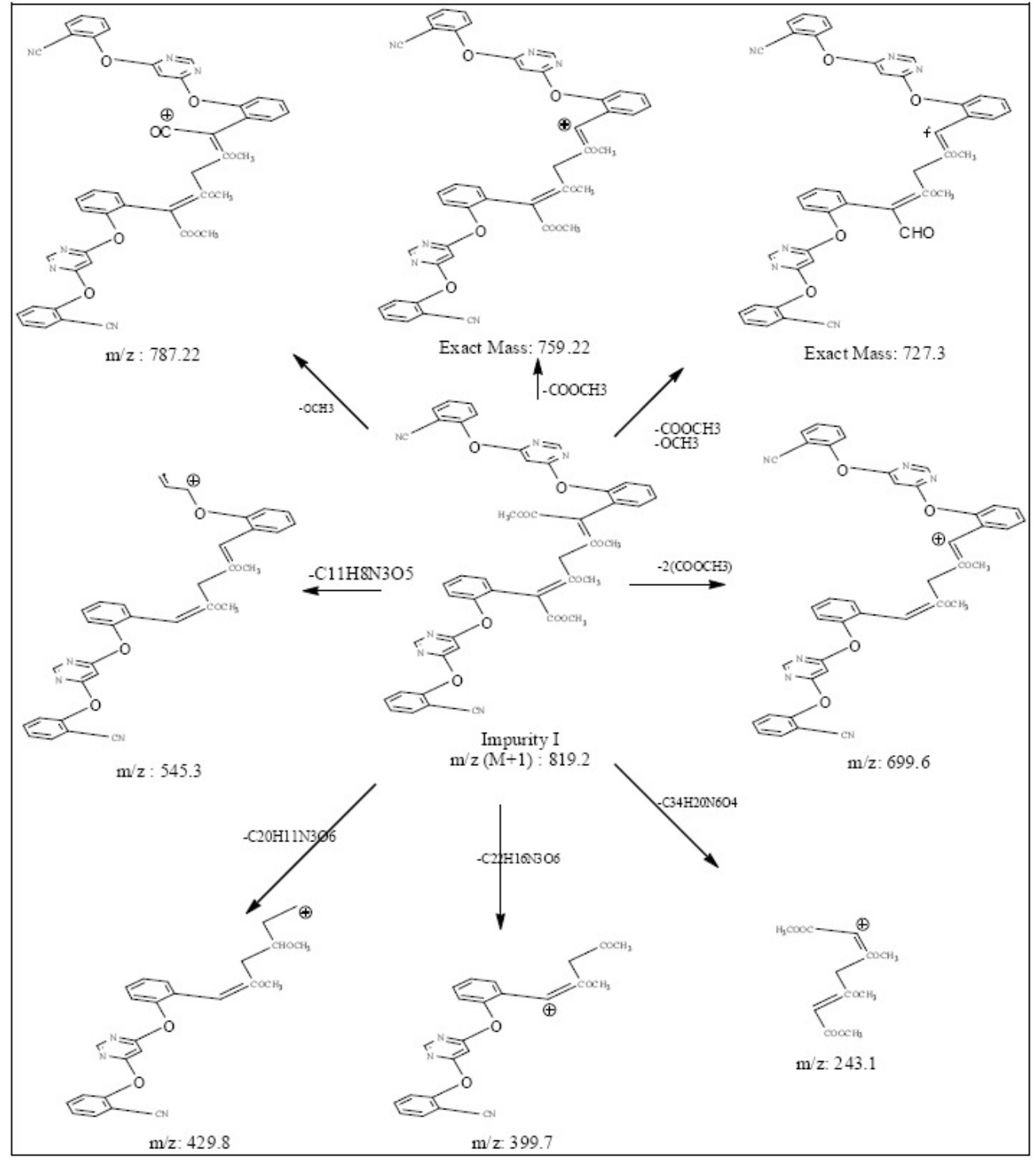

Figure 2. Mass fragmentation of impurity I

The mass fragmentation of impurity I supports to the chemical structure predicted in Figure 1. The ${ }^{1} \mathrm{H}$ NMR spectrum of the impurity I (Figure 5) exhibited signals corresponding to the aromatic protons (16 No's) are resonated at chemical shifts between $7.13 \mathrm{ppm}$ to $7.72 \mathrm{ppm}$, two protons corresponding to pyrimidine rings as a singlet at about 
$6.43 \mathrm{ppm}$ and at about $8.39 \mathrm{ppm}$, ethylene protons appeared at about $7.48 \mathrm{ppm}$, methylene protons appeared at about $4.06 \mathrm{ppm}$. The protons corresponding to two methoxy groups positioned, 1 alpha $-\mathrm{O}-\mathrm{C}=\mathrm{C}$ are resonated at about $3.63 \mathrm{ppm}$, and protons corresponding to two methoxy groups positioned 1 alpha $-\mathrm{OC}(=\mathrm{O})-\mathrm{C}=\mathrm{C}$ are appeared at about $3.82 \mathrm{ppm}$. The ${ }^{13} \mathrm{C}$ NMR spectrum of impurity I exhibited signals corresponding to the aromatic carbons (18 No's) and ethylene carbons (2 No's) are resonated at chemical shifts between $107 \mathrm{ppm}$ to $172 \mathrm{ppm}$, methylene protons appeared at about $40 \mathrm{ppm}$. The protons corresponding to two methyl groups positioned, 1 alpha $-\mathrm{O}-\mathrm{C}=\mathrm{C}$ are resonated at about 51.5 $\mathrm{ppm}$, and protons corresponding to two methyl groups positioned 1 alpha $-\mathrm{OC}(=\mathrm{O})-\mathrm{C}=\mathrm{C}$ are appeared at about $62 \mathrm{ppm}$. For the comparison, the ${ }^{1} \mathrm{H}$ NMR spectrum of azoxystrobin was shown in Figure 4.

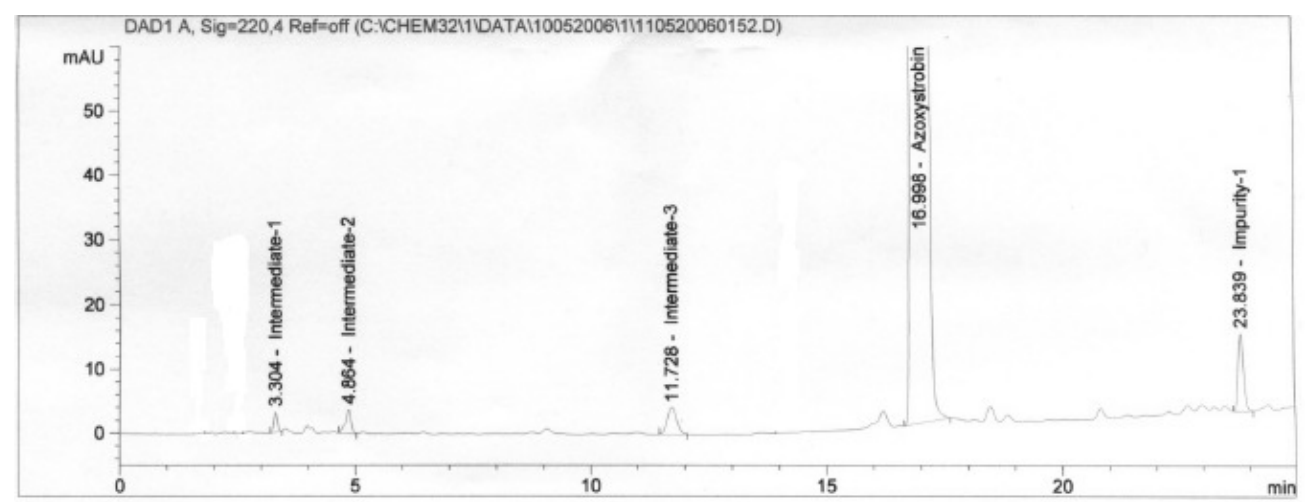

Figure 3. HPLC chromatogram of Azoxystrobin showing an impurity at $23.839 \mathrm{~min}$.

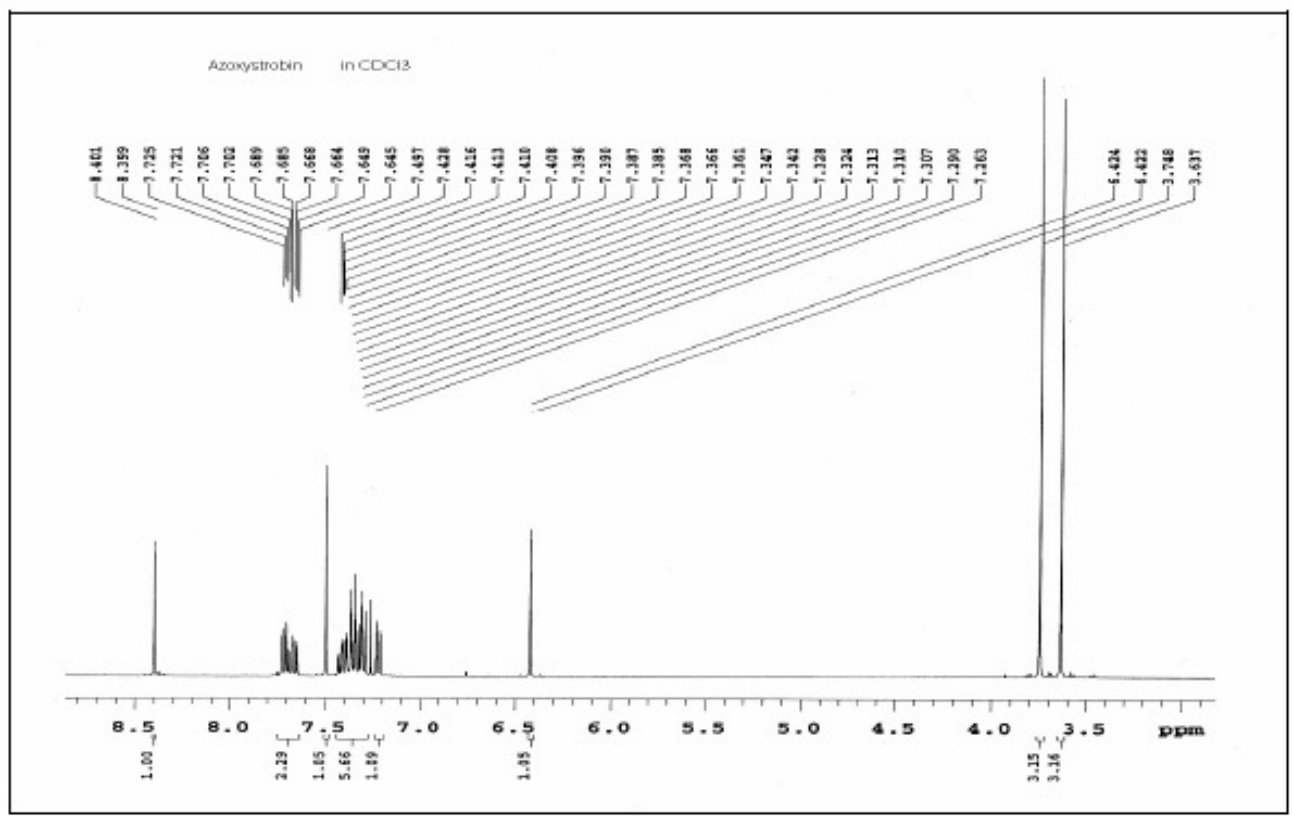

Figure 4. ${ }^{1} \mathrm{H}$ NMR spectrum of azoxystrobin 


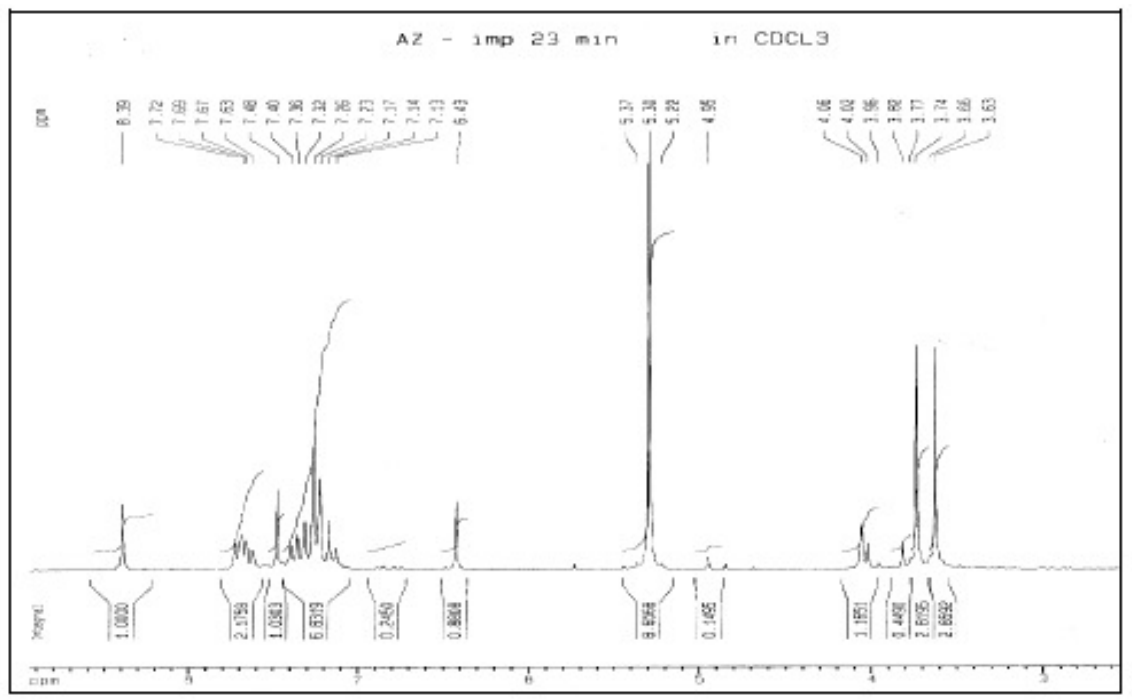

Figure 5. ${ }^{1} \mathrm{H}$ NMR spectrum of impurity I

Based on these spectral results, it was confirmed that the impurity having the molecular formula $\mathrm{C}_{45} \mathrm{H}_{34} \mathrm{~N}_{6} \mathrm{O}_{10}$ and the same was characterized as methyl 2-(2-(6-(2-cyanophenoxy)-2((4-(2-cyanophenoxy)-6-(2-(1,3-dimethoxy-3-oxoprop -1- en- 2- yl) phenoxy) pyrimidin -5- yl) methyl)pyrimidin-4-yloxy)phenyl)-3-methoxyacrylate.

\section{Formation of impurity $(\mathrm{m} / \mathrm{z}$ 819)}

During the penultimate stage of azoxystrobin synthetic process, dichloro methane was used as media to obtain azoxystrobin, which leads to the formation of this impurity during the final step of the synthesis of azoxystrobin.

\section{Conclusion}

This research paper describes the identification, isolation and structural elucidation of a process related impurity present in the azoxystrobin. The impurity was separated by reverse phase chromatographic technique, further isolated this impurity by semipreparative liquid chromatography. The isolated azoxystrobin impurity was characterized using spectroscopic techniques. This isolated impurity was used for calculating the mass balance of azoxystrobin technical material. The formation of the impurity was also discussed in brief.

\section{References}

1. Hou S C, Zhou Z Q, Qiao Z, Guo H C, Shi X Y, Wang M, Chromatographia, 2003, 57, 3-4, 177-180.

2. J M Clough, CRA Godfrey, et al, Azoxystrobin, A Novel Broad-spectrum Systemic Fungicide, Pesticide Outlook, 1996, 7:16-20.

3. CDS Tomlin Ed., The Pesticide Manual, British Crop Protection Council (BCPC) UK, 2000.

4. Gorog S, Identification and Determination of impurity in drugs, Elsevier, Amsterdam, 2000.

5. World Health Organisation Recommended Classification of Pesticides by Hazard 1998-99 (ref: WHO/PCS/98.2), WHO, Geneva 1999. 


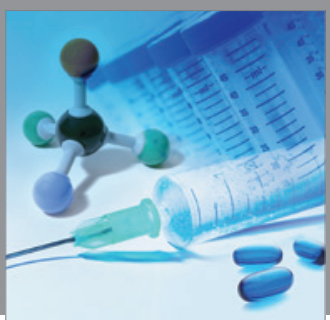

International Journal of

Medicinal Chemistry

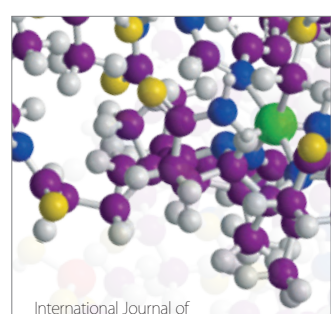

Carbohydrate Chemistry

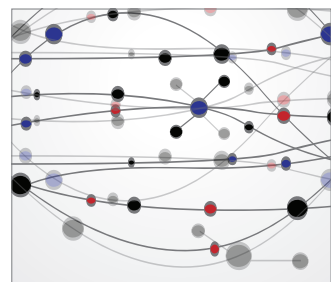

The Scientific World Journal
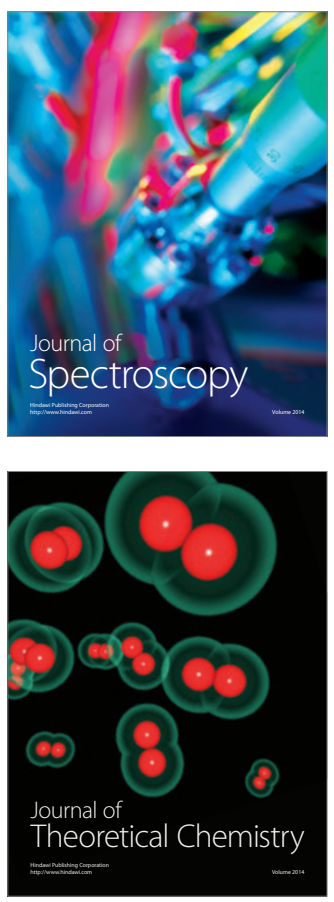
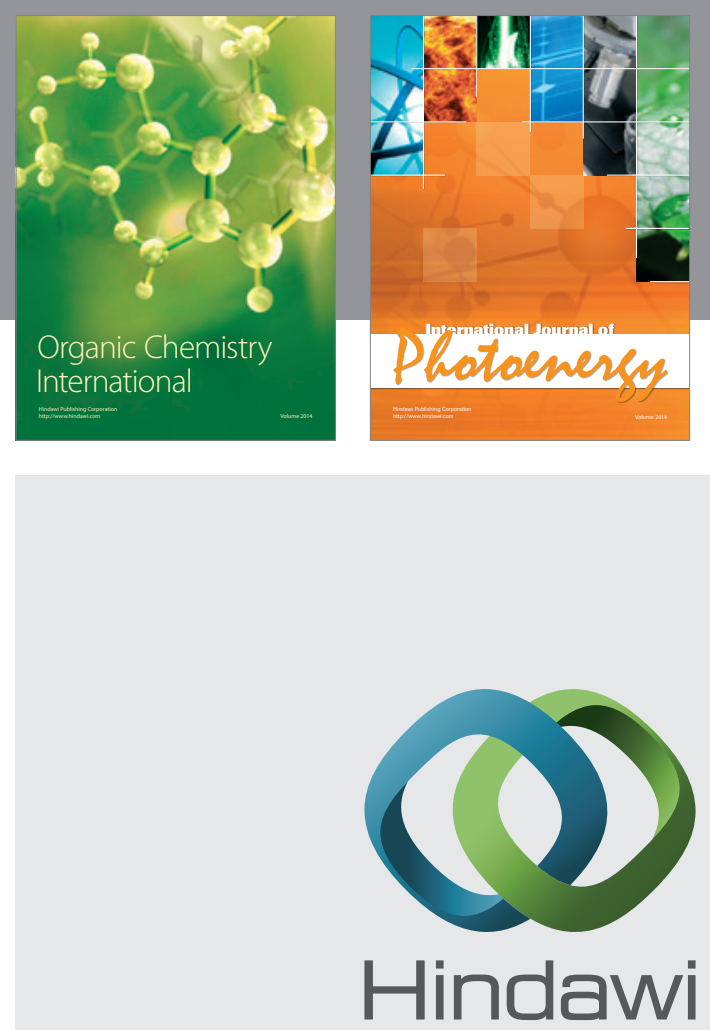

Submit your manuscripts at

http://www.hindawi.com
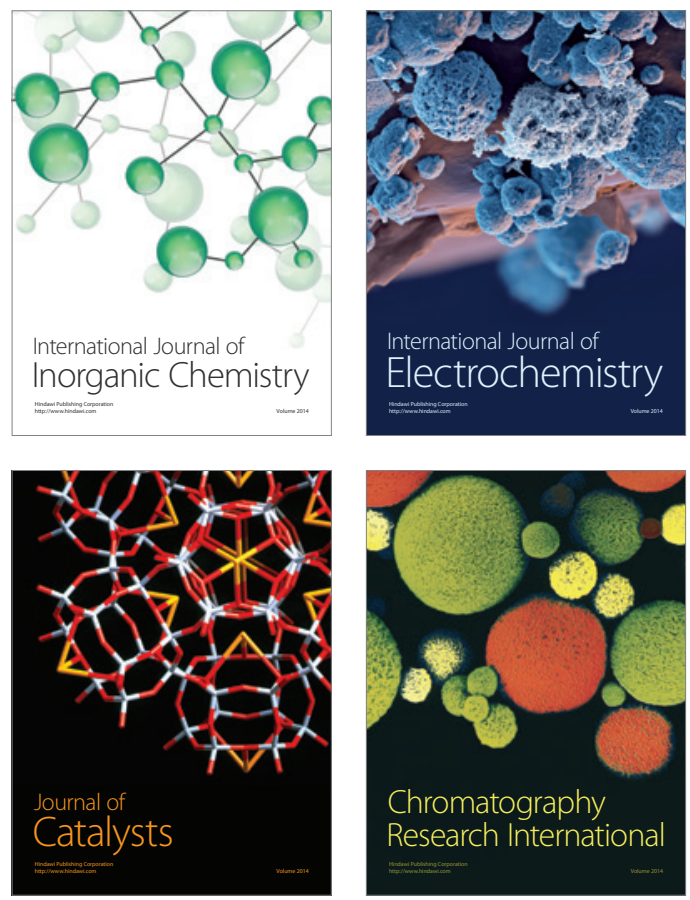
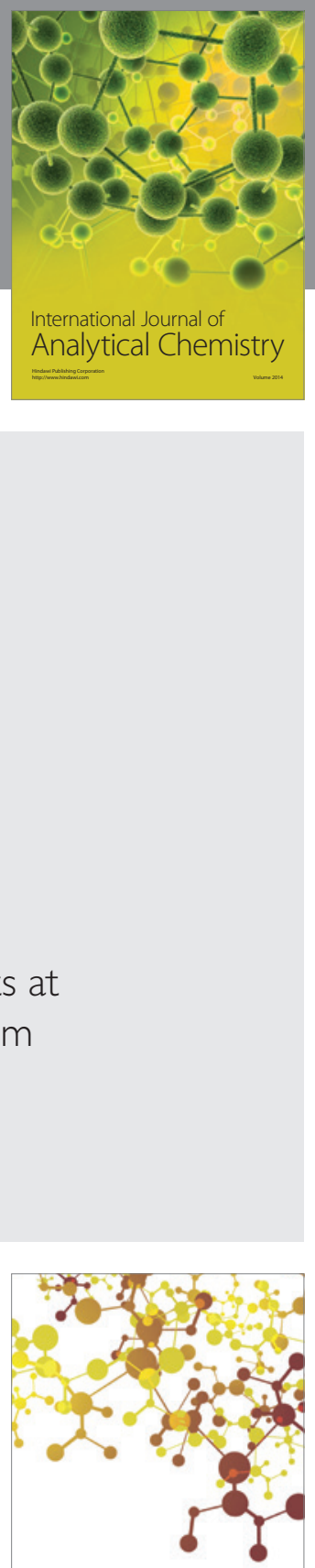

Journal of

Applied Chemistry
